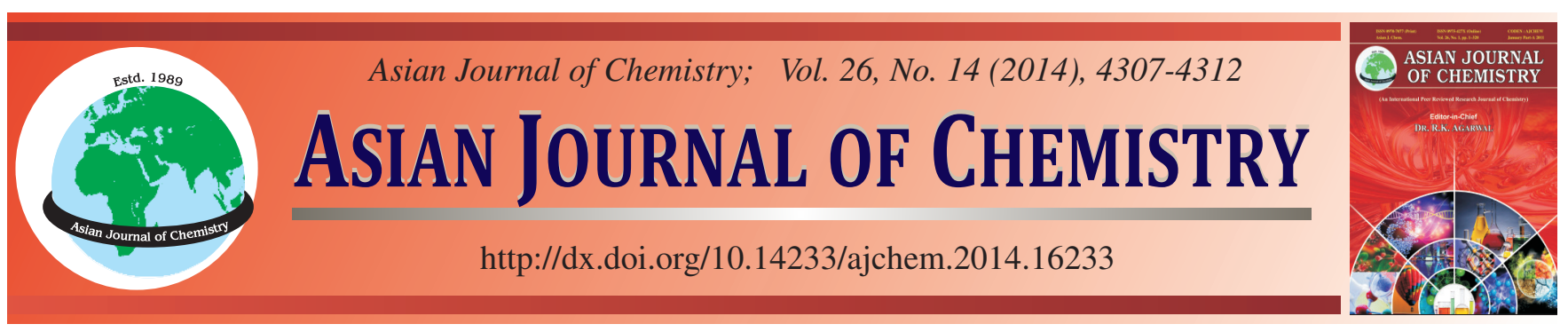

\title{
Synthesis and Enhanced Properties of Fluorescence-Based Polyurethane Films by Covalently Bonding 1,8-Naphthalimide
}

\author{
Y. MA, Q.Y. TANG, L.H. WANG and C. YAO*
}

School of Science, Nanjing University of Technology, Nanjing 211816, P.R. China

*Corresponding author: Tel/Fax: +86 25 58139482; E-mail: yaochengnjut@126.com

Received: 24 August 2013;

Accepted: 27 October 2013;

Published online: 5 July 2014;

AJC-15464

\begin{abstract}
A series of fluorescence-based polyurethane films (AABD-PUs) were synthesized by covalently bonding different amounts of 2-(2aminoethyl)-6-((2-aminoethyl)amino)-1H-benzo[de]isoquino-line-1,3(2H)-dione (AABD) into polyurethane chains as a chain extender, which was a novel amino-functionalized 1,8-naphthalimide fluorescent dye. The chemical structure of AABD was confirmed by ${ }^{1} \mathrm{H}$ NMR and FT-IR spectra. The AABD segments content in synthesized AABD-PUs was investigated by ultraviolet visible spectroscopy (UVvisible) and the enhanced properties of AABD-PUs were characterized by fluorescent spectroscopy. It was found that the UV-visible absorption and the fluorescence emission maximum of AABD-PUs showed blue shift in comparison with those of AABD, respectively. The fluorescence intensity of AABD-PUs was observably enhanced due to the changes of micro-circumstance of AABD. Furthermore, the fluorescence of AABD-PUs showed quite good stability, not only for the temperature but also for the fluorescence quencher. Additionally, the fluorophore was fixed permanently into polyurethane chains and was difficult to migrate.
\end{abstract}

Keywords: Fluorescence, Polyurethane, Film, 1,8-Naphthalimide.

\section{INTRODUCTION}

Recently, fluorescence-based polymers ${ }^{1-3}$ have attracted great attention due to their intrinsic advantages including excellent biocompatibility, low toxicity, long-term stability and facile conjugation with functional molecules. In particular, polyurethane is one of the most popular polymers based on its enormous diversity of chemical compositions and properties ${ }^{4-6}$. The fluorescence-based polyurethane can be applied not only in textiles, moldings, films, coating materials and paper making, but also in organic light emitting diodes, medical devices and fluorescent probes $^{7-10}$.

1,8-Naphthalimide derivatives are interesting compounds due to their useful photophysical and biological properties that offer promise for medical applications as free radical scavengers, potential photoredox anticancer agents, fluorescent labels, photosensitizers and imaging agents ${ }^{11-14}$. Additionally, they are particularly attractive as fluorophores because they can demonstrate high quantum yields, good photostability and their fluorescence can be tuned throughout a wide spectral range from blue to red ${ }^{15}$. The attractive properties of the 1,8 naphthalimide fluorophore have led to its incorporation into numerous polymers so as to tailor solubility, self-association and molecular size to suit a particular application ${ }^{16,17}$.

However, the direct application of 1,8-naphthalimide derivatives is unfavorable, as it is difficult to immobilization, easy to wash off and not resistant to solvent. As a result, the design of inserting the fluorophore of organic fluorescent dyes into polymer chains has attracted a considerable attention ${ }^{18,19}$. Chemical anchoring of small organic fluorescent dyes into polymer matrixes to afford fluorescence-based polymers has been motivated because of the enhanced solvent resistance, resistance to migration and better processability ${ }^{20-22}$. Organic fluorescent dyes conjugated into polymer chains through covalent bonds could enhance their photostability and fluorescence quantum yield. But, conventional organic fluorescent dyes are difficult to react with 4,4'-methylenediphenyl diisocyanate (MDI, or other diisocyanates) with the presence of other additives to form fluorescence-based polyurethane. Besides, organic fluorescent dyes are not uniformly dispersed or dissolved in normal resin materials, so novel fluorescent dyes with more reactive groups need to be developed.

In this work, 4-bromo-1,8-naphthalic anhydride was chosen as a starting material to react with ethylene diamine to form a novel amino-functionalized fluorescent dye, 2-(2-aminoethyl)6-((2-aminoethyl)amino)-1H-benzo[de]isoquino-line-1,3(2H)dione (AABD). Fluorescence-based polyurethane films (AABDPUs) were successfully synthesized by attaching different amounts of AABD into polyurethane chains as a chain extender via a two-step process. The AABD segments content in synthesized AABD-PUs was investigated by ultraviolet visible spectroscopy (UV-visible) and the enhanced properties of AABD-PUs were 
characterized by fluorescent spectroscopy. In addition, the thermal migration property of AABD-PUs was also studied.

\section{EXPERIMENTAL}

4-Bromo-1,8-naphthalic anhydride, rhodamine $6 \mathrm{G}$ and 4, 4'-methylenediphenyl diisocyanate (MDI) were received from Aldrich chemical Company and MDI was vacuum distilled before use. Polypropylene glycol (PPG, Mn = 1000) was desiccated at $110^{\circ} \mathrm{C}$ before use, which was supplied by Zhongshan Chemical Co., Ltd (Nanjing, China). Trimethylolpropane (TMP), N-methyl-2-pyrrolidone (NMP), ethylene diamine, hydroquinone and acetone were all reagent grade and purchased from Sinopharm Chemical Reagent Co., Ltd (Beijing, China).

Synthesis of AABD: The synthesis of AABD is shown in Scheme-I. 4-Bromo-1,8-naphthalic anhydride (2 g, $7.22 \mathrm{mmol})$ was dissolved in ethanol with the presence of ethylene diamine $(50 \mathrm{~mL})$ and the resulting solution was heated under reflux for $12 \mathrm{~h}$. The solution was allowed to cool and the ethylene diamine was distilled under vacuum to afford a crude product. The pure compound AABD was obtained after silica gel chromatography using dichloromethane as eluent.

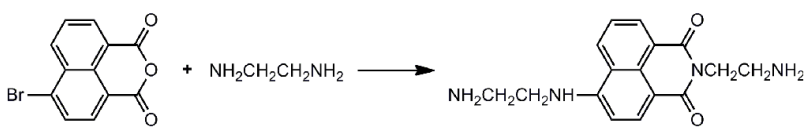

Scheme-I: Synthesis of AABD

Synthesis of AABD-PUs: A series of AABD-PUs were prepared by two-step bulk polymerization without catalyst, as shown in Scheme-II. All glassware was dried in an oven overnight at $105{ }^{\circ} \mathrm{C}$.
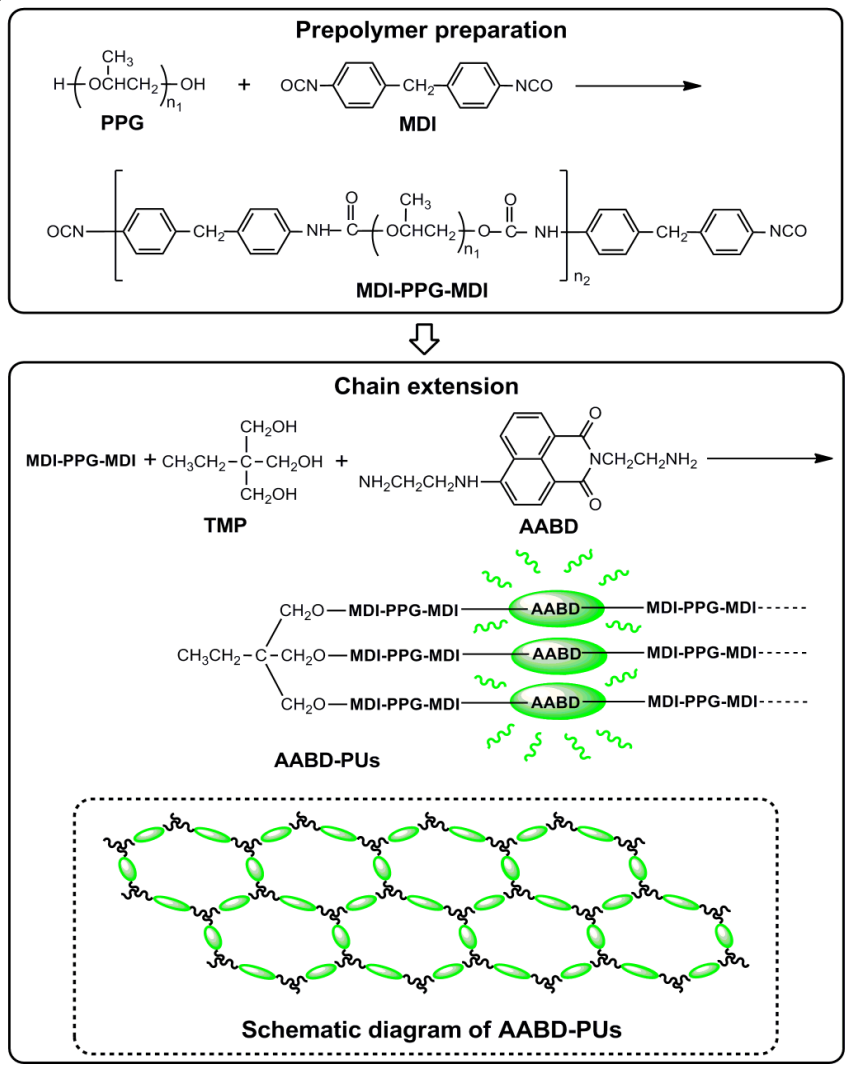

Scheme-II: Preparation of AABD-PUs
Prepolymer preparation: Polypropylene glycol (5 g, $5 \mathrm{mmol}$ ) was charged in a $100 \mathrm{~mL}$ four-necked flask equipped with a mechanical stirrer, a nitrogen inlet, a reflux condenser and a thermometer. MDI (2.82 g, $11.27 \mathrm{mmol})$ was dripped down in 30-60 min controlling the temperature below $40{ }^{\circ} \mathrm{C}$. Then the mixture reacted at $80{ }^{\circ} \mathrm{C}$ for $2 \mathrm{~h}$ under $\mathrm{N}_{2}$ to obtain an isocyanate-terminated polymer having an isocyanate content of 6.5 wt. $\%$, which was quantitatively determined by the din-butylamine method ${ }^{23}$.

Chain extension: Following the above-mentioned process, suitable amount of acetone was added into the system to reduce the viscosity. Firstly, AABD with a stoichiometric ratio as listed in Table- 1 was added in the reactor at $80^{\circ} \mathrm{C}$ and stirred vigorously for $2 \mathrm{~min}$. Subsequently, TMP was added and stirred vigorously for $2 \mathrm{~min}$, too. Then the reactor was distilled in vacuo to ensure the removal of air bubbles and partial acetone. Finally, the mixture was cast in a preheated teflon mould to form a 100-200 $\mu \mathrm{m}$ film and cured for $24 \mathrm{~h}$ at $60{ }^{\circ} \mathrm{C}$.

\begin{tabular}{ccccc}
\multicolumn{5}{c}{ TABLE-1 } \\
\multicolumn{5}{c}{ COMPOSITIONS OF POLYURETHANE } \\
FILMS PREPARED IN THIS STUDY \\
\hline Sample & MDI $(\mathrm{g})$ & PPG $(\mathrm{g})$ & TMP $(\mathrm{g})$ & AABD $(\mathrm{g})$ \\
\hline PU & 2.82 & 5.00 & 0.515 & 0 \\
AABD-PU1 & 2.82 & 5.00 & 0.463 & 0.171 \\
AABD-PU2 & 2.82 & 5.00 & 0.411 & 0.343 \\
AABD-PU3 & 2.82 & 5.00 & 0.360 & 0.514 \\
AABD-PU4 & 2.82 & 5.00 & 0.309 & 0.685 \\
\hline
\end{tabular}

${ }^{1} \mathrm{H}$ NMR spectra of AABD was measured on a Bruker AV-400 spectrometer with chemical shifts reported in ppm (in DMSO- $d_{6}$; TMS as internal standard). FT-IR spectrum of AABD was measured by a FT-IR spectrometer (Nicolet 8700 , Boston, MA) in the wave number of $4000-400 \mathrm{~cm}^{-1}$ at $25^{\circ} \mathrm{C}$. $\mathrm{UV}$-visible spectra and fluorescence spectra were recorded on a Perkin-Elmer 35 spectrometer and a Perkin-Elmer LS 50B fluorescence spectrophotometer at room temperature, respectively.

\section{RESULTS AND DISCUSSION}

Characterization of AABD: Fig. 1 shows the typical ${ }^{1} \mathrm{H}$ NMR spectra of AABD. Yield $91 \%$; m.p. $234.4-235.7{ }^{\circ} \mathrm{C} ;{ }^{1} \mathrm{H}$ NMR (400 MHz, DMSO- $\left.d_{6}\right): \delta(\mathrm{ppm})=8.52-8.55(\mathrm{~d}, J=8.72$ $\mathrm{Hz}, 1 \mathrm{H}, \mathrm{Ar}-\mathrm{H}), 8.32-8.34$ (d, $J=7.16 \mathrm{~Hz}, 1 \mathrm{H}, \mathrm{Ar}-\mathrm{H}), 8.09-$ $8.11(\mathrm{~d}, J=8.28 \mathrm{~Hz}, 1 \mathrm{H}, \mathrm{Ar}-\mathrm{H}), 7.60-7.64(\mathrm{t}, J=7.48 \mathrm{~Hz}, 1 \mathrm{H}$, Ar-H), 6.73-6.75 (d, $J=8.44 \mathrm{~Hz}, 1 \mathrm{H}, \mathrm{Ar}-\mathrm{H}), 4.83$ (s, 4H, $-\mathrm{NH}_{2}$ ), 4.41 (s, 1H, -NH-), 4.04-4.07 (t, $J=6.20 \mathrm{~Hz}, 2 \mathrm{H},-\mathrm{CH}_{2}-$ ), $3.93-3.96\left(\mathrm{t}, J=5.24 \mathrm{~Hz}, 2 \mathrm{H},-\mathrm{CH}_{2}-\right), 3.22-3.25(\mathrm{t}, J=5.24$ $\left.\mathrm{Hz}, 2 \mathrm{H},-\mathrm{CH}_{2^{-}}\right), 2.99-3.02\left(\mathrm{t}, J=6.20 \mathrm{~Hz}, 2 \mathrm{H},-\mathrm{CH}_{2^{-}}\right)$. Anal. Calcd for $\mathrm{C}_{16} \mathrm{H}_{18} \mathrm{~N}_{4} \mathrm{O}_{2}$ : C, 64.41; H, 6.08; N, 18.78. Found: C, 64.49; H, 6.04; N, 18.71. TOF-MS: $m / z 299.34[\mathrm{M}+1]^{+}$.

The FT-IR spectra for 4-bromo-1,8-naphthalic anhydride and AABD are given in Fig. 2. The presence of AABD was demonstrated by the formation of the absorbance peaks at around $3400 \mathrm{~cm}^{-1}[\mathrm{v}(\mathrm{N}-\mathrm{H})], 2900 \mathrm{~cm}^{-1}[\mathrm{v}(\mathrm{C}-\mathrm{H})], 1646 \mathrm{~cm}^{-1}$ $[\mathrm{v}(\mathrm{C}=\mathrm{O})], 1576 \mathrm{~cm}^{-1}[\delta(\mathrm{N}-\mathrm{H})], 1412 \mathrm{~cm}^{-1}[\delta(\mathrm{C}-\mathrm{H})]$ and 1363 $\mathrm{cm}^{-1}[\mathrm{v}(\mathrm{C}-\mathrm{N})]$. Additionally, the absorbance peak at $557 \mathrm{~cm}^{-1}$ $[\mathrm{v}(\mathrm{C}-\mathrm{Br})]$ almost totally disappeared in comparison to that of 4-bromo-1,8-naphthalic anhydride. These results shown in 
Figs. 1 and 2 confirmed that the reaction of 4-bromo-1,8naphthalic anhydride with ethylene diamine with the presence of ethanol to form a novel amino-functionalized fluorescent dye, AABD, did take place.

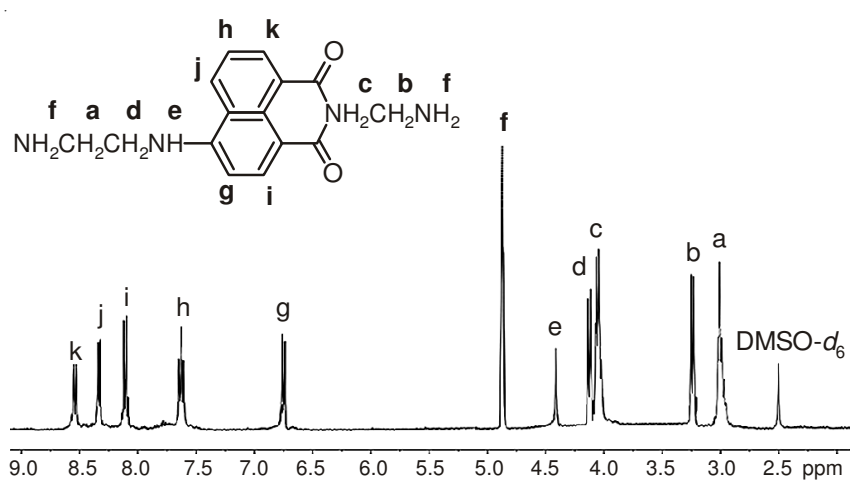

Fig. 1. ${ }^{1} \mathrm{H}$ NMR spectra of AABD

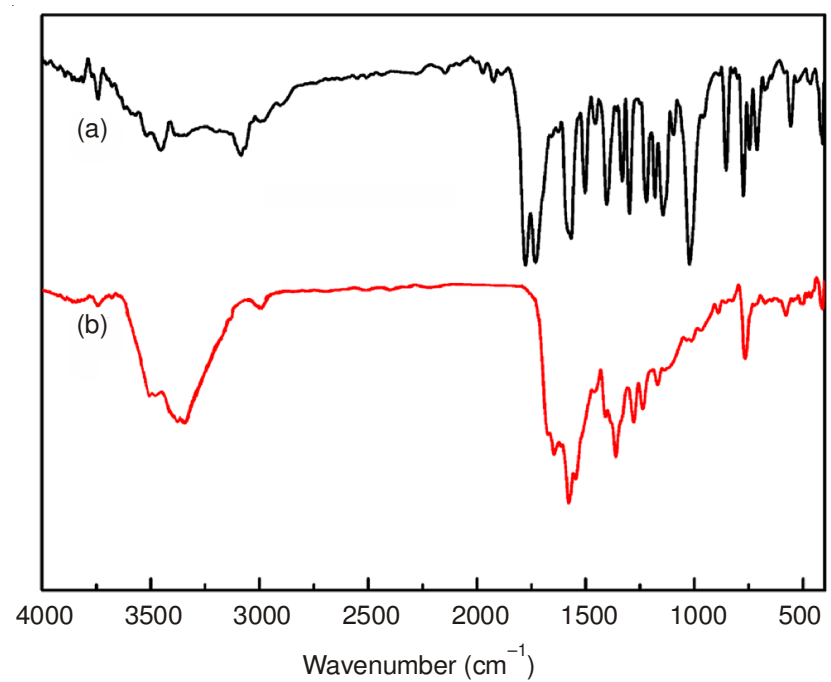

Fig. 2. FT-IR spectra of (a) 4-bromo-1,8-naphthalic anhydride, (b) AABD

UV-visible absorption: In this study, UV-visible spectroscopy was used to measure the AABD segments content in synthesized polyurethane films. In order to obtain the standard curve, firstly, AABD was dissolved in NMP solution with different concentrations. The UV-visible absorbance spectra of AABD with different concentrations are shown in Fig. $3 \mathrm{a}$. We could obtain the interaction between the maximum absorbance peak value and the AABD concentration in the inset of Fig. 3a. As a result, the fitting curve equation could be obtained for $\mathrm{y}=0.07738 \mathrm{x}-0.00142(\mathrm{x}: \mathrm{AABD}$ concentration; $\mathrm{y}$ : the maximum absorbance peak value).

Then we measured the UV-visible absorbance spectra of AABD-PUs in the same solvent. Fig. 3 b shows the UV-visible spectra of the common polyurethane (PU) and AABD-PUs in NMP. Herein, the weight percentage of AABD segments in AABD-PU1, AABD-PU2, AABD-PU3 and AABD-PU4 were determined by the curve equation listed in Table-2. From the data, it could be seen that experimental values of AABD content was very close to the design values, which further confirmed that AABD was entirely conjugated into polyurethane chains. Moreover, the UV-visible spectra of AABD-PUs showed an absorption peak at $413 \mathrm{~nm}$ due to the covalent bonding with AABD in comparison with that of PU without conjugating AABD. The absorption maximum of AABD-PUs and AABD were 413 and $426 \mathrm{~nm}$, respectively. AABD-PUs showed blue shift about $13 \mathrm{~nm}$, due to the $\pi-\pi^{*}$ transition caused by the conjugation between carbonyl and the naphthalene ring.
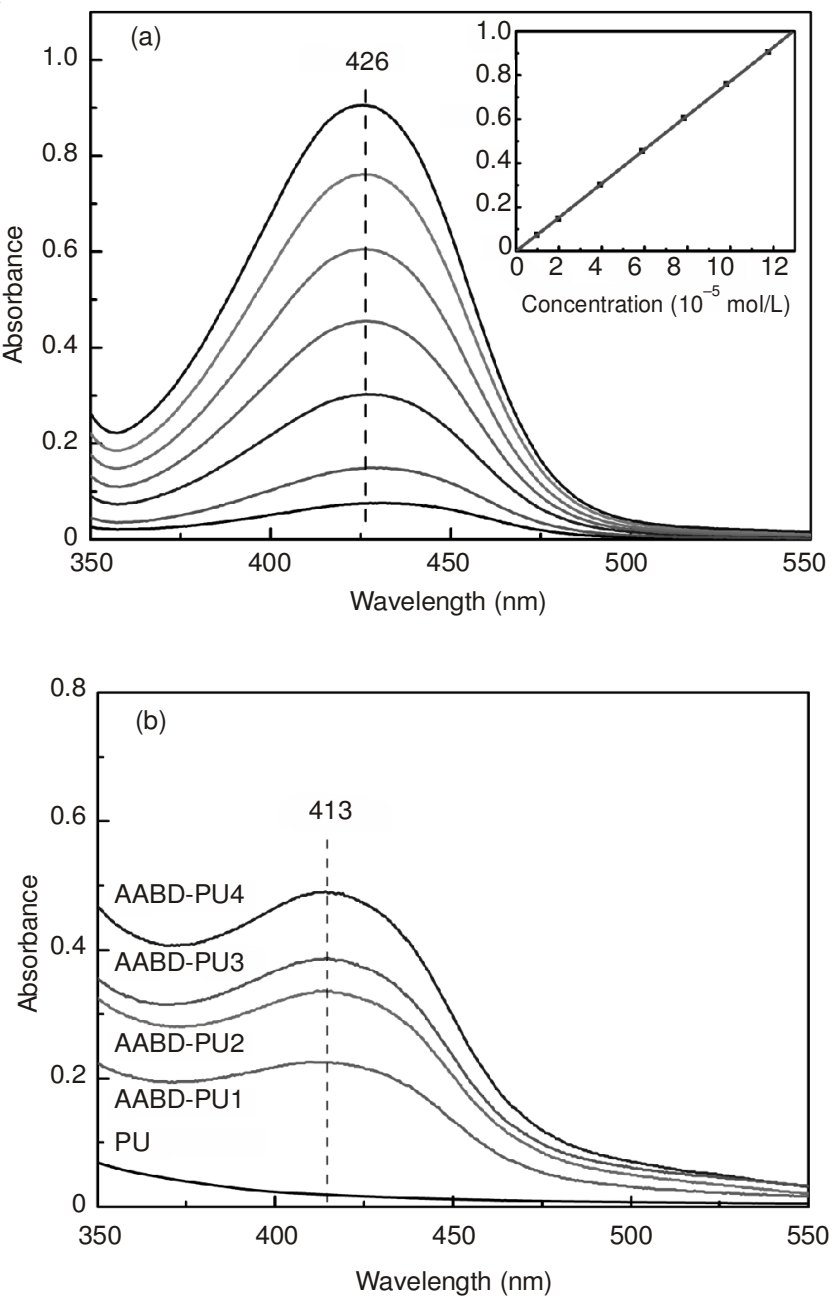

Fig. 3. (a) UV-visible absorbance spectra with different concentrations of AABD in NMP. Inset: a linear relationship curve of the maximum absorbance peak value versus the AABD concentration. (b) UVvisible absorption spectra of polyurethane (PU) and AABD-PUs in NMP

TABLE-2

CONTENT OF AABD SEGMENTS IN THE SYNTHESIZED AABD-PUS

\begin{tabular}{ccccc} 
Sample & weight $(\mathrm{g})$ & Maximum absorption value & AABD (wt. \%, experimental value) & AABD (wt. \%, design value) \\
AABD-PU1 & 0.440 & 0.225 & 1.98 & 2.03 \\
AABD-PU2 & 0.321 & 0.334 & 4.03 & 3.99 \\
AABD-PU3 & 0.251 & 0.386 & 5.94 & 5.91 \\
AABD-PU4 & 0.242 & 0.489 & 7.81 & 7.76 \\
\hline
\end{tabular}


Enhanced fluorescent properties of AABD-PUs: To evaluate the enhanced properties of AABD-PUs, the fluorescent spectroscopy were measured. Due to the shape of fluorescence emission spectra of AABD-PUs were similar and had the same maximum emission wavelength, as a representative, fluorescence spectra of AABD-PU2 was chosen to study the fluorescent properties. As shown in Fig. 4a, the fluorescence intensity of AABD-PU2 in NMP was higher than that of AABD with the same fluorophore concentration. Compared with AABD in NMP, the emission maximum of AABD-PU2 in NMP showed blue shift about $10 \mathrm{~nm}$, while that of AABD-PU2 film showed blue shift about $22 \mathrm{~nm}$. It was found that the emission maximum of AABD-PU2 film showed blue shift $12 \mathrm{~nm}$ in comparison with that of AABD-PU2 in NMP, which might be ascribed to the stabilization of the excited state of fluorophores when AABDPU2 was dissolved in NMP solution. Correspondingly, as shown in Fig. 4b, the images of polyurethane (PU) and AABD-PU2 films were taken under daylight and a $365 \mathrm{~nm}$ ultraviolet lamp, respectively. AABD-PU2 displayed bright green color and intense fluorescence compared with polyurethane. In contrast, polyurethane without conjugating AABD showed no light emission within the range of $400 \mathrm{~nm}$ to $700 \mathrm{~nm}$.
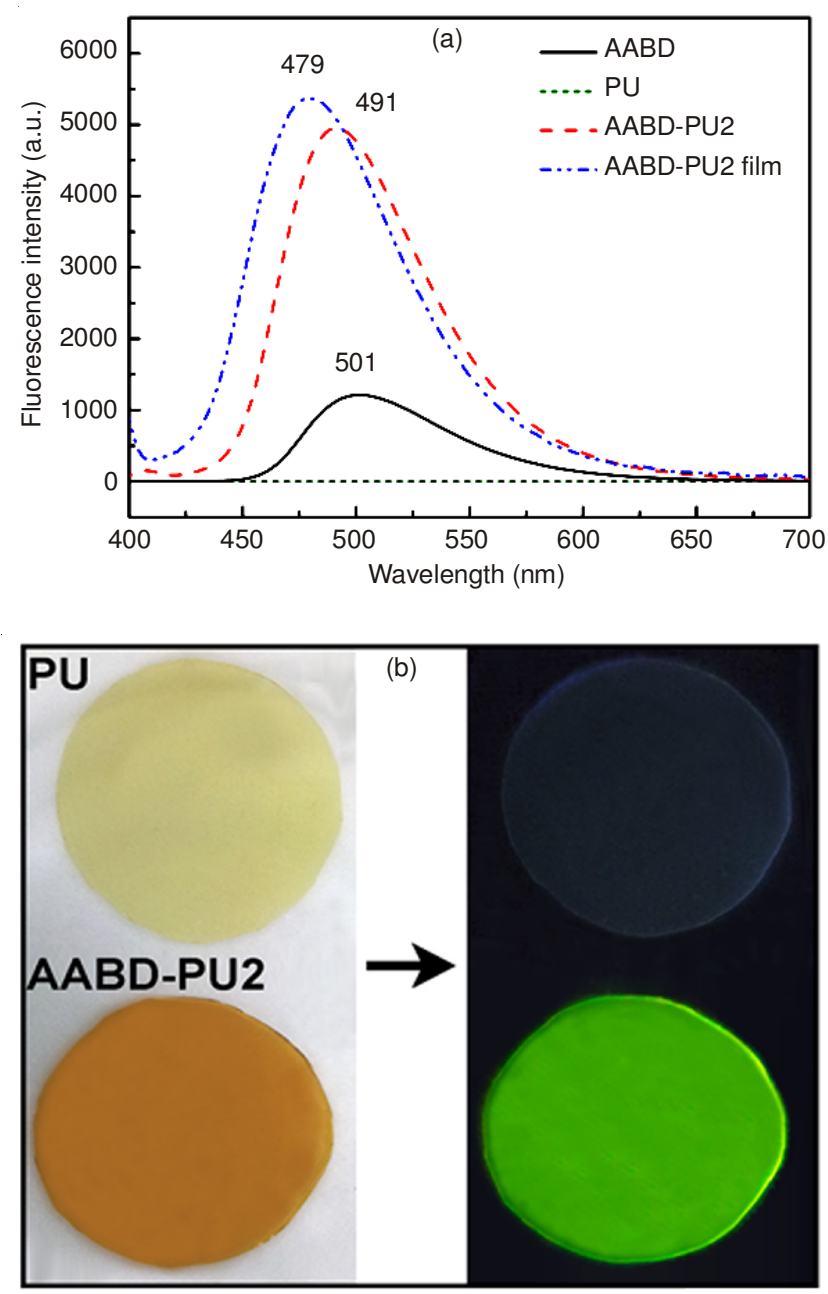

Fig. 4. (a) Fluorescence emission spectra of polyurethane (PU), AABD, AABD-PU2 in NMP and AABD-PU2 film. AABD and AABD-PU2 in NMP with the same fluorophore concentration of $2 \times 10^{-4} \mathrm{~mol} / \mathrm{L}$, $\lambda_{\mathrm{ex}}=415 \mathrm{~nm}$. (b) The optical images of polyurethane and AABDPU2 taken under daylight and a $365 \mathrm{~nm}$ UV lamp, respectively
It is well known that Stoke's shift $(\Delta v)$ and fluorescence quantum yield are important parameters for fluorescent dyes. The Stoke's shift $(\Delta v)$ can demonstrate the difference in structure and property of fluorescent dyes between the first excited state $S_{1}$ and the ground state $S_{0}$. The Stoke's shift could be calculated according to the following equation ${ }^{16}$ :

$$
\Delta v=\frac{\left(\lambda_{\mathrm{F}}-\lambda_{\mathrm{A}}\right) \times 10^{7}}{\lambda_{\mathrm{F}} \lambda_{\mathrm{A}}}
$$

where $\lambda_{\mathrm{A}}$ was the UV-visible maximum absorption of AABDPU2 $(413 \mathrm{~nm})$ and $\lambda_{\mathrm{F}}$ was the fluorescent maximum emission of AABD-PU2 (491 nm). The Stoke's shift of $3846 \mathrm{~cm}^{-1}$ was determined, which was indicative of energy loss between the excitation and the emission state, as the excited molecules experienced vibrational relaxation or internal conversion, as well as the solvation effect.

Moreover, the fluorescence quantum yield was used to evaluate the ability of fluorescent dyes to emit the absorbed light energy. The fluorescence quantum yield of AABD and AABD-PU2 in NMP was determined, using rhodamine $6 \mathrm{G}$ as the standard. The quantum yield of AABD and AABD-PU2 could be calculated using the equation ${ }^{18}$ :

$$
\mathrm{Y}_{\mathrm{u}}=\mathrm{Y}_{\mathrm{s}} \times \frac{\mathrm{F}_{\mathrm{u}}}{\mathrm{F}_{\mathrm{s}}} \times \frac{\mathrm{A}_{\mathrm{s}}}{\mathrm{A}_{\mathrm{u}}}
$$

where $Y_{s}$ was the quantum yield of rhodamine $6 \mathrm{G}$ as a standard (the quantum yield $=0.95$ ), $\mathrm{Y}_{\mathrm{u}}$ was that of the unknown sample to be measured; $F_{\mathrm{s}}$ was the integrated fluorescence intensity of rhodamine $6 \mathrm{G}$ and $\mathrm{F}_{\mathrm{u}}$ was that of the unknown sample; As was the absorbance intensity of excitation wavelength for rhodamine $6 \mathrm{G}$ and $\mathrm{A}_{\mathrm{u}}$ was that for the unknown sample. The quantum yield of AABD and AABD-PU2 was 0.13 and 0.57 , respectively. The quantum yield of AABD-PU2 was 4 times higher than that of AABD in NMP. These results suggested that the fluorescence performance was better for AABD-PU2 than for $\mathrm{AABD}$, as a result of the changes of micro-circumstance of AABD. Since AABD was chemically bonded into polyurethane chains, the less exciplex formation among the fluorophores resulted in the enhanced fluorescence intensity. On the other hand, the relatively low fluorescence intensity for $\mathrm{AABD}$ was attributed to fluorescence quenching named "structural self-quenching"24.

To understand if the "concentrational self-quenching" existed in AABD-PUs, the fluorescence emission spectra of AABD and AABD-PU2 in a broad concentration range of $1 \times$ $10^{-6} \mathrm{~mol} / \mathrm{L}$ to $1 \times 10^{-3} \mathrm{~mol} / \mathrm{L}$ were recorded, respectively. As shown in Fig. 5, it was interesting to note that fluorescence intensity of AABD-PU2 was always higher than that of AABD at different concentrations. Further, the fluorescence intensity of both AABD and AABD-PU2 increased first as the concentration increased and then decreased when the concentration was beyond $2 \times 10^{-4} \mathrm{~mol} / \mathrm{L}$. It was confirmed that "concentrational self-quenching" existed in both AABD and AABD-PU2 through aggregation of the fluorophore ${ }^{25}$.

In addition, the fluorescence stability of AABD-PUs was also investigated. The fluorescence spectra of AABD and AABD-PU2 with the same fluorophore concentration under different temperatures are shown in Fig. 6. The fluorescence intensity of AABD and AABD-PU2 decreased with the growing 


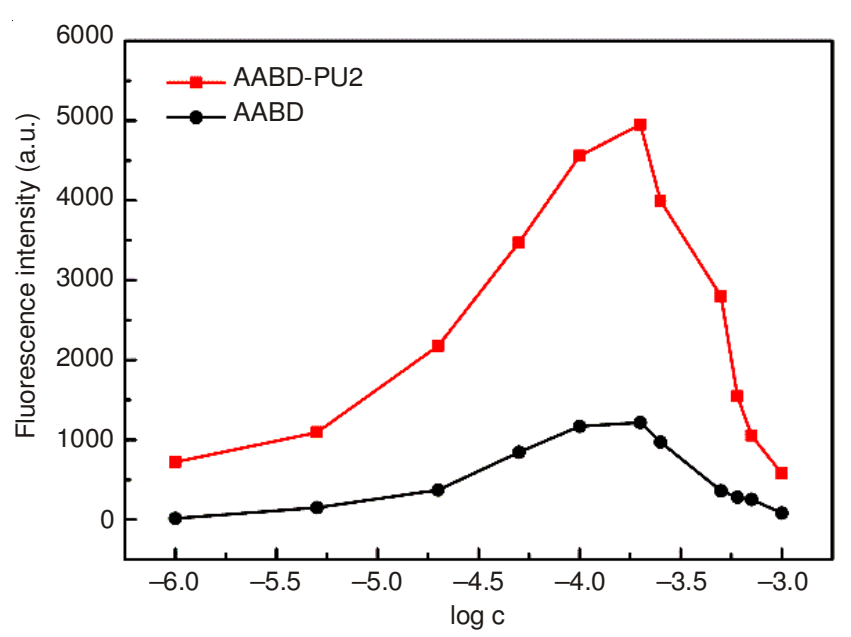

Fig. 5. Fluorescence intensity of AABD-PU and AABD-PU2 in NMP in a broad concentration range

temperature because of the effect of energy transform. It was also found that AABD in NMP was more sensitive to temperature changes than AABD-PU2. Moreover, the fluorescence of AABD-PUs showed quite good stability during storage. After one month placed at ambient temperature, hardly any difference of fluorescence spectra was observed.
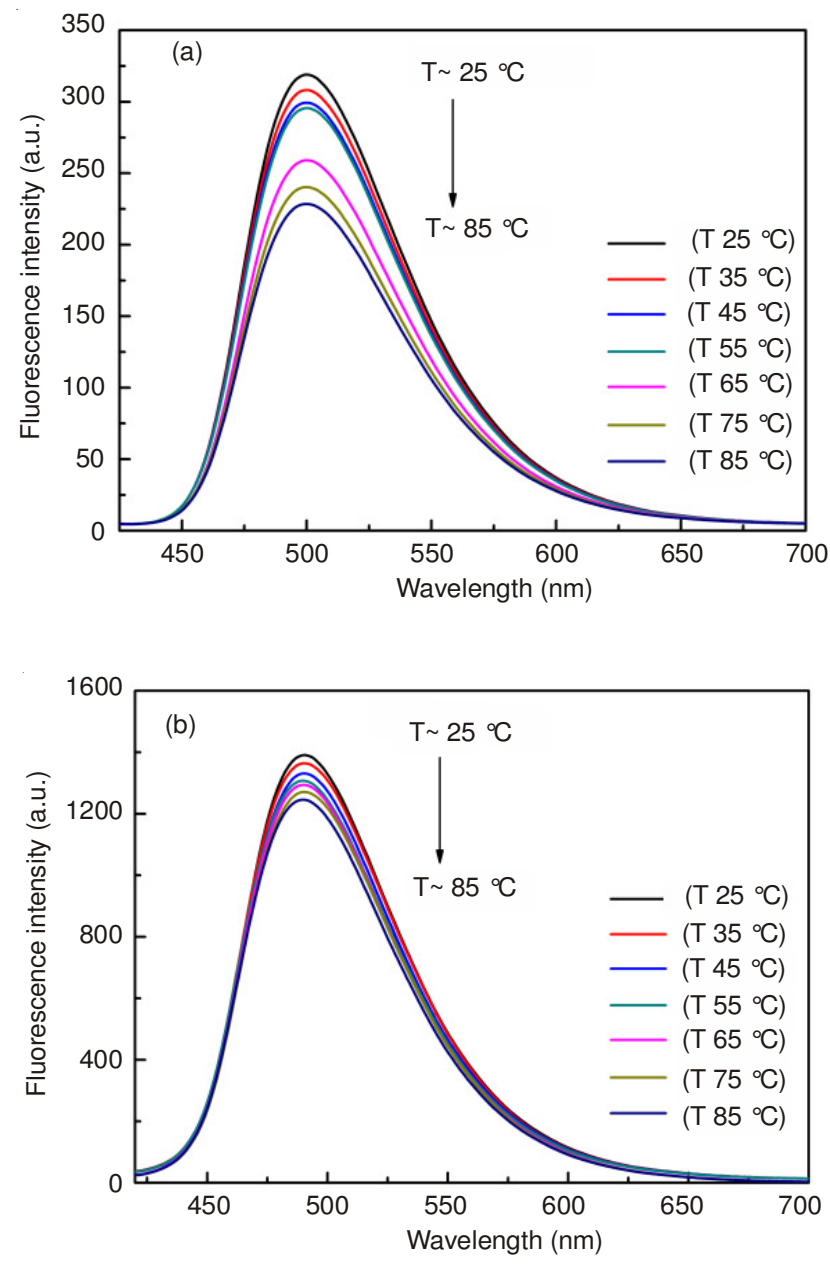

Fig. 6. Fluorescence spectra of (a) AABD, (b) AABD-PU2 under different temperature $\left(\lambda_{\mathrm{ex}}=415 \mathrm{~nm}\right.$, concentration of AABD segments $=1.5$ $\times 10^{-5} \mathrm{~mol} / \mathrm{L}$
The fluorescence stability of AABD-PU2 can also be confirmed by a fluorescence quencher. According to the literature method $^{26-28}$, hydroquinone was generally used as a quencher to analyze the fluorescence stability. Fig. 7 shows the fluorescent spectra of AABD-PU2 in NMP with hydroquinone as a quencher. The fluorescence intensity of AABD-PU2 could be regularly quenched by adding the hydroquinone. The fluorescence quenching efficiency could be calculated according to the Stern-Volmer equation:

$$
\frac{\mathrm{I}_{0}}{\mathrm{I}_{\mathrm{Q}}}=1+\mathrm{K}_{\mathrm{SV}}[\mathrm{Q}]
$$

where $\mathrm{I}_{0}$ was the fluorescence intensity of AABD-PU2 in NMP without hydroquinone, while $\mathrm{I}_{\mathrm{Q}}$ was the fluorescence intensity with the presence of hydroquinone; [Q] was the concentration of hydroquinone in NMP and $\mathrm{K}_{\mathrm{SV}}$ was the Stern-Volmer constant. $K_{\text {Sv }}$ value indicated the fluorescence quenching efficiency. The data shown in Fig. 7 present a straight line nearly passing through the point $(0,1)$. Finally, a very small dynamic quenching constant $\mathrm{K}_{\mathrm{Sv}}$ of $2.01 \mathrm{M}^{-1}$ could be determined from the slope of the line. Overall, the fluorescence of AABD-PUs was not sensitive to the quencher, too.

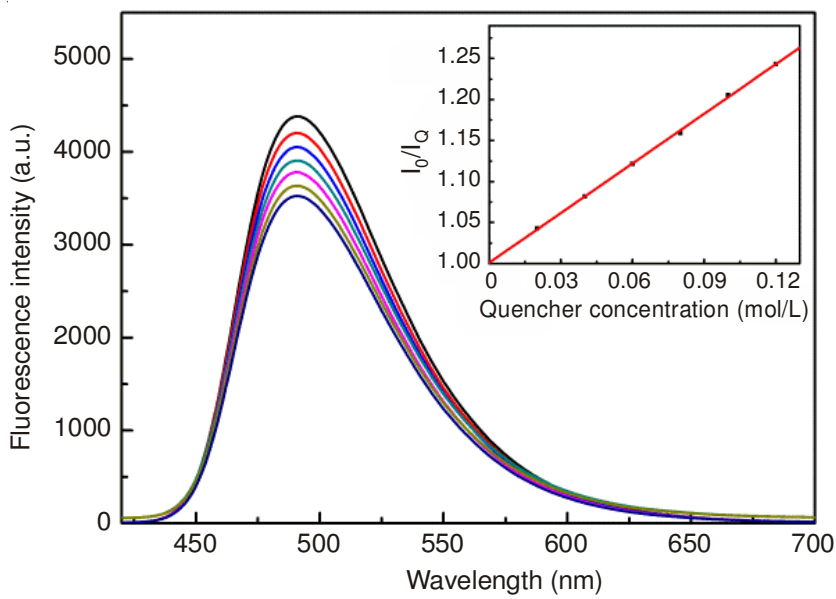

Fig. 7. Fluorescence spectra of AABD-PU2 quenched by hydroquinone. Inset: the plot of $\mathrm{I}_{0} / \mathrm{I}_{\mathrm{Q}}$ versus the concentration of hydroquinone [Q]

Thermal migration property of AABD-PUs: Generally speaking, AATCC ${ }^{29}$ method was used to test the migration property for the fluorescent dyes. In order to assess the migration property of AABD-PUs, firstly, a composite sample of AABD + PU film was prepared using the blend of PU with $\mathrm{AABD}$ and the AABD content in AABD + PU was 4 wt. \%, same as the AABD content of AABD-PU2. As a reference, AABD-PU2 was also chosen to study the thermal migration property. The polyurethane film was covered with a $60 \mathrm{~mm}$ diameter watch (region A). After keeping the temperature at $60{ }^{\circ} \mathrm{C}$ for $24 \mathrm{~h}$, the same weight of region A's film and uncovered film (region B) were dissolved in NMP, then measured UVvisible absorption of the solution. The migration property (MP) could be calculated using the equation:

$$
\mathrm{M}_{\mathrm{P}}=\frac{\mathrm{C}_{\mathrm{B}}-\mathrm{C}_{\mathrm{A}}}{\mathrm{C}_{\mathrm{A}}} \times 100 \%
$$

where $C_{A}$ and $C_{B}$ were the fluorophore content of region $A$ and $\mathrm{B}$, respectively. Table- 3 lists the parameters of fluorophore 
migration property for AABD + PU and AABD-PU2 films. It was noteworthy that the $\mathrm{M}_{\mathrm{P}}$ of AABD-PU2 was only $1.02 \%$, while that of AABD + PU was $24.6 \%$. The results showed that the fluorophore $\mathrm{M}_{\mathrm{P}}$ in AABD-PU2 was less than that in the composite AABD + PU. As expected, the advantage of using the novel amino-functionalized fluorescent dye over conventional dyes was that the dye became chemically bonded (-NH-CO-NH-) into polyurethane chains. Thus, the fluorophore of AABD-PUs was difficult to migrate.

\begin{tabular}{cccc}
\hline \multicolumn{5}{c}{ TABLE-3 } \\
PARAMETERS OF THERMAL MIGRATION \\
PROPERTY IN POLYURETHANE FILMS \\
\hline Sample & $\mathrm{C}_{\mathrm{A}}(\%)$ & $\mathrm{C}_{\mathrm{B}}(\%)$ & $\mathrm{M}_{\mathrm{P}}(\%)$ \\
\hline AABD + PU & 3.01 & 3.75 & 24.6 \\
AABD-PU2 & 3.93 & 3.97 & 1.02 \\
\hline
\end{tabular}

\section{Conclusion}

In summary, a series of fluorescence-based polyurethane films (AABD-PUs) were successfully synthesized by attaching different amounts of AABD into polyurethane chains as a chain extender, which was a novel amino-functionalized 1,8-naphthalimide fluorescent dye. The chemical structure of AABD was confirmed by ${ }^{1} \mathrm{H}$ NMR and FT-IR spectra. The AABD segments content in synthesized AABD-PUs was investigated by UVvisible spectra and the enhanced properties of AABD-PUs were characterized by fluorescent spectroscopy. Compared with AABD in NMP, the UV-visible absorption maximum of AABDPUs showed blue shift about $13 \mathrm{~nm}$, while the fluorescence emission maximum of AABD-PU2 in solution and film state showed blue shift about $10 \mathrm{~nm}$ and $22 \mathrm{~nm}$, respectively. The fluorescence intensity of AABD-PUs was observably enhanced due to the changes of micro-circumstance of AABD. The quantum yield of AABD-PUs in NMP was 4 times higher than that of AABD. Furthermore, the fluorescence of AABD-PUs showed quite good stability, not only for the temperature but also for the fluorescence quencher. Additionally, the fluorophore of AABD-PUs was difficult to migrate and MP of AABD-PU2 was only $1.02 \%$.

\section{ACKNOWLEDGEMENTS}

Financial support from the 863 program (2011AA02A204) is acknowledged.

\section{REFERENCES}

1. J.H. Kim, K. Park, H.Y. Nam, S. Lee, K. Kim and I.C. Kwon, Prog. Polym. Sci., 32, 1031 (2007).

2. T. Klingstedt and K.P.R. Nilsson, Biochim. Biophys. Acta, 1810, 286 (2011).

3. H.Y. Yang, X.M. Zhang, L.J. Duan, M.Y. Zhang, G.H. Gao and H.X. Zhang, J. Polym. Res., 19, 9973 (2012).

4. C.W. Meuse, X.Z. Yang, D.C. Yang and S.L. Hsu, Macromolecules, 25, 925 (1992).

5. K. Kojio, Y. Mitsui and M. Furukawa, Polymer, 50, 3693 (2009).

6. Z.D. Shi and X.L. Wang, Polym. Adv. Technol., 20, 1017 (2009).

7. H.N. Kim, Z.Q. Guo, W.H. Zhu, J. Yoon and H. Tian, Chem. Soc. Rev., 40, 79 (2010).

8. V.B. Bojinov and D.B. Simeonov, Polym. Degrad. Stab., 95, 43 (2010).

9. M.O. Liu, H.-F. Lin, M.-C. Yang, M.-J. Lai, C.-C. Chang, H.-C. Liu, P.-L. Shiao, I.-M. Chen and J.-Y. Chen, Mater. Lett., 60, 2132 (2006).

10. L. Torini, J.F. Argillier and N. Zydowicz, Macromolecules, 38, 3225 (2005).

11. O. Jaudouin, J.J. Robin, J.M. Lopez-Cuesta, D. Perrin and C. Imbert, Polym. Int., 61, 495 (2012).

12. Y. Zhang, S. Feng, Q. Wu, K. Wang, X. Yi, H. Wang and Y. Pan, Med. Chem. Res., 20, 752 (2011).

13. J.E. Rogers and L.A. Kelly, J. Am. Chem. Soc., 121, 3854 (1999).

14. A.M.M. El-Betany and N.B. McKeown, Tetrahedron Lett., 53, 808 (2012).

15. H.B. Xiao, M.J. Chen, G.H. Shi, L. Wang, H.Y. Yin and C. Mei, Res. Chem. Intermed., 36, 1021 (2010).

16. I. Grabchev, S. Sali, R. Betcheva and V. Gregoriou, Eur. Polym. J., 43, 4297 (2007).

17. I. Grabchev and V. Bojinov, Polym. Degrad. Stab., 70, 147 (2000).

18. N.Z. Galunov, B.M. Krasovitskii, O.N. Lyubenko, I.G. Yermolenko, L.D. Patsenker and A.O. Doroshenko, J. Lumin., 102-103, 119 (2003).

19. X.H. Hu, X.Y. Zhang, J.B. Dai and J. Liu, J. Lumin., 131, 2160 (2011).

20. Y.C. Chen, R.R. Chiou, H.L. Huang, Y.Z. Tsai, Y.M. Kuo and D.Y. Chao, J. Appl. Polym. Sci., 97, 455 (2005).

21. Y.Y. Zhang and C.H. Zhou, Bioorg. Med. Chem. Lett., 21, 4349 (2011).

22. Y. Wang, X. Zhang, B. Han, J. Peng, S. Hou, Y. Huang, H. Sun, M. Xie and Z. Lu, Dyes Pigments, 86, 190 (2010).

23. D.J. David and H.B. Staley, Analytical Chemistry of the Polyurethanes, Wiley-Interscience, New York, Vol. 16 (1969).

24. F.M. Li, S.J. Chen, Z.C. Li and J. Qiu, J. Polym. Sci. Polym. Chem., 34, 1881 (1996).

25. J. Qiu, Z.C. Li, Q.Y. Gao, G.-Q. Yao, G.-X. Yang, J.-X. Zhang and F.M. Li, J. Polym. Sci. Polym. Chem., 34, 3015 (1996).

26. L. Biczok and H. Linschitz, J. Phys. Chem., 99, 1843 (1995).

27. C. Turro, S.H. Bossmann, Y. Jenkins, J.K. Barton and N.J. Turro, J. Am. Chem. Soc., 117, 9026 (1995).

28. J. McCall, C. Alexander and M.M. Richter, Anal. Chem., 71, 2523 (1999).

29. A.A.T.C.C. Test Method, 140-2001, Dye and Pigment Migration in a PadDry Process, In: AATCC Technical Manual, American Association of Textile Chemists and Colorists (2006). 